\title{
Factors That Influence Changes to Existing Chronic Pain Management Plans
}

\author{
Julie Diiulio, MS, Laura G. Militello, MA, Barbara T. Andraka-Christou, JD, PhD, \\ Robert L. Cook, MD, MPH, Robert W. Hurley, MD, PhD, Sarah M. Downs, MPH, \\ Shilo Anders, PhD, Burke W. Mamlin, MD, Elizabeth C. Danielson, MA, and \\ Christopher A. Harle, PhD
}

Background: The objective of this qualitative study is to better understand primary care clinician decision making for managing chronic pain. Specifically, we focus on the factors that influence changes to existing chronic pain management plans. Limitations in guidelines and training leave clinicians to use their own judgment and experience in managing the complexities associated with treating patients with chronic pain. This study provides insight into those judgments based on clinicians' first-person accounts. Insights gleaned from this study could inspire innovations aimed at supporting primary care clinicians (PCCs) in managing chronic pain.

Methods: We conducted 89 interviews with PCCs to obtain their first-person perspective of the factors that influenced changes in treatment plans for their patients. Interview transcripts were analyzed thematically by a multidisciplinary team of clinicians, cognitive scientists, and public health researchers.

Results: Seven themes emerged through our analysis of factors that influenced a change in chronic pain management: 1) change in patient condition; 2) outcomes related to treatment; 3 ) nonadherent patient behavior; 4) insurance constraints; 5) change in guidelines, laws, or policies; 6) approaches to new patients; and 7) specialist recommendations.

Conclusions: Our analysis sheds light on the factors that lead PCCs to change treatment plans for patients with chronic pain. An understanding of these factors can inform the types of innovations needed to support PCCs in providing chronic pain care. We highlight key insights from our analysis and offer ideas for potential practice innovations. (J Am Board Fam Med 2020;33:42-50.)

Keywords: Chronic Pain, Clinical Decision Making, Opioids, Pain Management, Primary Health Care, Qualitative Research

Up to 100 million adults in the United States are affected by chronic pain, leading to an economic cost that exceeds that of heart disease. ${ }^{1}$ Due to an

This article was externally peer reviewed.

Submitted 10 August 2019; revised 20 October 2019; accepted 21 October 2019.

From Applied Decision Science, LLC, Dayton, OH (JD, LGM); Department of Health Management \& Informatics, University of Central Florida, Orlando, FL (BTAC); University of Florida Gainesville, FL (RLC); Department of Anesthesiology and Public Health Science, Wake Forest University School of Medicine, Winston Salem, NC (RWH); Department of Health Policy and Management, Indiana University Richard M. Fairbanks School of Public Health, Indianapolis, IN (SMD, ECD, CAH); Department of Anesthesiology, Vanderbilt University Medical Center, Nashville, TN (SA); Department of Internal Medicine, Indiana University School of Medicine, Indianapolis, IN (BWM); Center for Biomedical Informatics, Regenstrief Institute, Indianapolis, IN (BWM, CAH). undersupply of specialist pain management physicians, primary care clinicians (PCCs) are mainly responsible for pain management care for chronic

Funding: This project was supported by Grant R01HS023306 from the Agency for Healthcare Research and Quality, Designing User-Centered Decision Support Tools for Primary Care Pain Management. The content is solely the responsibility of the authors and does not necessarily represent the official views of the Agency for Healthcare Research and Quality.

Conflict of interest: CAH is a principal investigator on past and current research grants from the Agency for Healthcare Research and Quality (AHRQ) and Pfizer, Inc. related to chronic pain care. RC receives funding from the National Institutes of Health (NIH) and the Florida Department of Health. RWH receives funding from AHRQ, NIH, Medtronic (educational grant to Wake Forest University School of Medicine), Medtronic (consultancy), and the Robert Wood Johnson Foundation (research funding as a Clinical Scholar). BTAC serves as a coinvestigator on an NIH 
noncancer pain. ${ }^{2,3}$ Despite their important role in managing pain, PCCs have limited training in pain management. ${ }^{3,4}$ Even with training, chronic pain management is complex due to its biopsychosocial nature, etiologic ambiguity, lack of effective treatments, and limited PCC time..$^{2,4-7}$ Several best practice guidelines have been created to assist clinicians in treating pain. ${ }^{8-10}$ However, studies have demonstrated a low level of guideline adherence for a variety of reasons. ${ }^{10-15}$ Furthermore, little is known about how interventions should be tailored for individual patients rather than for the average patient. $^{6}$

Limitations in guidelines and training leave PCCs to use their own judgment and experience in managing the complexities associated with treating patients with chronic pain. Several studies have looked at the effects of demographic factors on opioid prescribing for chronic pain. ${ }^{10,16-21}$ Chen et $\mathrm{al}^{17}$ indicated that a patient's specific pain diagnosis plays an important role in prescribing opioids. Turk and Okifuji ${ }^{21}$ found that behavioral manifestations of pain, especially nonverbal communication, and reports of functional disability, were important factors in pain management decisions. However, to our knowledge, few studies have examined the factors that lead PCCs to change already existing chronic pain management plans.

Our study extends prior research by employing naturalistic methods to explore PCC decision making for managing chronic pain. We use concrete examples of specific patients, described from the PCC's first-person perspective, to elucidate the factors that influence changes to existing chronic pain management plans. Understanding these factors is important because they can be used to ideate innovations aimed at supporting PCCs on the front lines of chronic pain care.

\section{Methods}

This method section describes a qualitative study in which a sample of 20 PCCs took part in a series of

R01 examining effects of state policies on pain management. The authors, JD, LGM, SMD, SA, ECD, and BWM declare that they have no affiliations with or involvement in any organization or entity with any financial interest in the subject matter or materials discussed in this paper.

Corresponding author: Julie Diiulio, MS, Applied Decision Science, 5335 Far Hills Ave \#103, Dayton, OH 45429 (E-mail: j.diiulio@applieddecisionscience.com). interviews about 1 or more of their patients with chronic pain. Patients did not participate in the interviews, but they were asked to consent to the use of their records during the interviews. The research team performed a thematic analysis of the interview transcripts, with a focus on the events that led to changes in pain management plans. The study was approved by the Indiana University Institutional Review Board.

\section{Participants}

Our sample was purposive with the aim to recruit PCCs who were able to prescribe opioids and had patients with chronic noncancer pain. We sought to recruit PCCs from urban, suburban, and rural health clinics within different health systems in Indiana. Recruitment took place between April 2016 and July 2018. We initially obtained resources to conduct up to 5 interviews with each of 25 PCCs for a total of 125 interviews. Analysis began as interview transcripts became available. The research team observed thematic saturation and stopped data collection after analyzing 89 interviews with 20 PCCs. Some PCCs participated in fewer than 5 interviews due to limited availability to participate or because thematic saturation had been reached and data collection stopped. A member of the study team, a public health researcher, recruited the participants through word-of-mouth, e-mail invitations, and in-person presentations at clinic staff meetings. Participants were given a $\$ 100$ gift card for each interview.

To identify the patients who would be discussed in the interviews, we worked with the PCCs. Typically, an interview would be scheduled, and then the provider would look at their patient schedule to find a patient who fit the eligibility criteria and had an upcoming appointment within 3 days before the scheduled interview. Patients were eligible if they were over 18 years, had chronic pain, and had no history of cancer within the past 3 years. To obtain patient permission to be used as the subject of the interview, a member of the research team met with eligible patients immediately before their appointments, confirmed eligibility, and invited the patients to participate in the study. Patients who agreed to be the subject of the PCC interview provided written informed consent and received a \$25 gift card. 


\section{Interviews}

Each interview focused on a unique patient with chronic noncancer pain. Interviews lasted approximately 60 minutes and took place in the PCCs' clinics. The interviewers included public health researchers and cognitive scientists from the research team. Three interviewers were present for the first 6 interviews. This was done to ensure common ground across the team and to facilitate refining the interview guide. The remaining interviews were conducted by 1 or 2 interviewers. The interviews occurred within 3 days following the patient appointment to facilitate accurate recall. PCCs had access to the patient's electronic medical record during the interview as a memory aid. All interviews were audio recorded and transcribed. Resulting transcriptions were deidentified.

At the initial interview, PCCs completed a demographic questionnaire and answered questions about their use of tools and general approach to chronic pain care. For the remainder of the first interview, and all subsequent interviews, we used an adapted Critical Decision Method (CDM) technique $^{22}$ to explore the major events that occurred over the course of treating the patient's chronic pain. CDM is an approach to cognitive task analysis that is aimed at understanding cognitive aspects of work, specifically tacit aspects of expertise. During the interview, PCCs were encouraged to describe key information that influenced their decision making for each major event. Questions related to changes in the patient's condition, treatment plan, and goals (eg, What new information did you get during the visit that impacted your view of the patient or treatment plan?).

\section{Analysis}

We used an inductive thematic analysis approach, wherein we focused on exploring differences in interpretation of the data, and then built consensus. ${ }^{23}$ First, a multidisciplinary team (3 public health researchers, 2 cognitive scientists, 2 PCCs, and 1 pain specialist physician) reviewed 2 transcripts and highlighted themes of interest. The topics of interest were used to create a preliminary codebook. The codes were then applied to other transcripts, with codes modified and added inductively overtime if additional topics of interest emerged in the data. The final codebook included 55 codes. After the codebook was finalized, remaining transcripts were coded by 2 members of the team who met regularly to reach coding consensus. Agreement involved a negotiation process during which discrepancies were discussed and a final code was applied. The code, "change in pain management plan," is the subject of this article. We labeled transcript excerpts with this code if they included any change that occurred in the PCC's plan to manage the patient's pain. We chose to focus on this code because less is understood about the factors that influence changes to existing pain management plans as opposed to other clinical decisions in pain care, such as the decision to initiate an opioid prescription.

The code, "change in pain management plan," was applied to 217 transcript excerpts. For each excerpt, we identified the event or information that triggered the change and the resulting clinical action that was taken (eg, medication change, referral, etc.). Excerpts that did not have a clear precipitating event or resulting action were removed from the data set. We also removed excerpts that did not involve a change made by the interviewee. For example, some PCCs described actions taken by other providers. These excerpts were excluded because they did not reflect the interviewee's own decision making. In sum, 86 event and action pairs were identified. The event and action pairs were categorized by theme, resulting in 7 themes, as described in the results. The themes that emerged were vetted by the larger research team before finalization.

\section{Results \\ Sample Findings}

Our sample consisted of 20 PCCs (18 physicians and 2 nurse practitioners) who represented 13 clinics within 3 Indiana health care systems. Note, 1 PCC and 4 patients were in Illinois. The PCCs had an average of 14 years practicing post medical school (range, 2 to 34 years). Gender was balanced with 10 men and 10 women. Sixteen of the participants identified as white, 2 as black, and 2 as Asian. None of the participants identified as Hispanic or Latino.

\section{Analysis Findings}

Seven themes emerged through our analysis of factors that influenced PCCs to change chronic pain management plans. Below, we describe the themes and illustrate them with participant quotes. Many 
of the quotes we highlight involve opioids. We found these quotes particularly relevant given the current opioid crisis in the U.S. For context, half of the event and action pairs (43/86) included an action related to opioids (ie, start, switch, or change dose). Furthermore, most of the event and action pairs (77/86) included an action related to medications (ie, start, stop, switch, or change dose). About a third of the event and action pairs (28/86) included some type of nonmedication action (ie, referral). It is important to note that over half (44/86) of the event and action pairs included multiple actions (ie, stopping 1 medication, starting another, and ordering a referral).

\section{Change in Patient Condition}

A change in patient condition was the most common reason cited for changing a pain management plan. Over half (52 of 86) of the event and action pairs reveal changes stemming from events such as acute injuries, new contraindications, or changes in symptoms.

For acute injuries, changes to pain management plans typically included short courses of muscle relaxants or steroids, temporary increases in opioid medications, nonsteroidal anti-inflammatory drugs (NSAIDs), physical therapy referrals, and rest, ice, compression, and elevation. Similarly, recent surgeries tended to result in temporary increases in opioid medications.

New contraindications, such as reduced kidney function or increased blood pressure, typically resulted in the discontinuation of specific medications such as NSAIDs. In 1 example, a recent fall by an elderly patient prompted a PCC to reconsider sedating medications.

"I stopped her amitriptyline given these falls because that was something that she was not getting a lot of clinical improvement from but was another sedating agent."

\section{-PCC 12, Pt 44}

New symptoms typically led to exploration of pain etiology including new tests, imaging, and referrals to specialists. New symptoms also led to the addition of new medications. For example, numbness and tingling led to the addition of gabapentin and depression symptoms led to the addition of duloxetine.

"[I] suggested duloxetine to improve his pain control related to sleep and perhaps allow him to [avoid the] nighttime narcotic. After all, that decision is based on clinical suspicion and experience that there may be... some emotional component, after his spouse was gone." -PCC 24, Pt 94

Worsening pain associated with the progression of a chronic disease, resulted in the most varied changes in pain management. PCCs tended to try new medications and interventions in hopes of reducing pain and increasing function. In these cases, PCCs were also more likely to report gradually increasing the dose of opioids over time.

\section{Outcomes related to treatment}

Another common reason cited for changing a pain management plan related to the effects of treatment. Fifteen of the event and action pairs revealed changes resulting from side effects, ineffective treatment, and effective treatment.

Side effects typically resulted in the discontinuation of the suspected treatment. For example, gastric upset frequently led to the discontinuation of NSAIDs. When treatments were ineffective, PCCs tended to discontinue the treatment and try something new. For example, when a patient reported that cyclobenzaprine did not help, the PCC switched to pregabalin. When a patient reported that tramadol did not help, the PCC switched to hydrocodone. In another example, an aging patient with declining health was on a high dose of opioids and not improving. The lack of improvement prompted the PCC to decrease the opioid.

"So, at some point, I did start cutting her back because, I mean, I was at a point where it clearly was not working. And I felt like continuing it was not going to be, you know, in her best interest..."

-PCC 2, Pt 5

When treatments were effective, PCCs often reduced other, less desirable, medications. In 1 case, as epidurals were introduced and pain was reduced, opioids were then decreased. In another case, the patient's weight loss opened up a new treatment option (knee surgery), which was ultimately successful, and led to the reduction of opioids.

"She was on the higher dose of medicine and was having a lot of problems, and then when she had her knee replaced last year, in the immediate postoperative period she needed more, but after that, after about a month or 2 after her surgery, her right knee pain got significantly better."

-PCC 20, Pt 71 


\section{Nonadberent Patient Behavior}

Nonadherent patient behavior was also cited as a reason for changing a pain management plan. Nine of the event and action pairs revealed changes that were triggered by nonadherent patient behavior. In some examples, patients violated their opioid contracts by acquiring additional pain medication from other clinicians following surgery. In these cases, PCCs responded by reducing the opioid prescription and scheduling more frequent visits to monitor progress and discuss addiction. In some cases, such as when a patient used illicit drugs or acquired a legal substance illicitly, PCCs responded by weaning opioids or recommending treatment for substance abuse disorder.

"Then her substance abuse became clearly evident because she failed her regular urine [toxicology test]. [Opioids] were slowly weaned off."

-PCC 3, Pt 7

"And then [she] did finally admit a couple of weeks after I initially met her that she had been buying them off the street. So, we got her into our Suboxone program and started her on Suboxone at that point. Which is for addiction, not so much for her pain, but... with her it is really all kind of mixed in with mental health and it is a complicated picture."

-PCC 22, Pt 88

In another case, a patient reported giving his opioids to his wife who was in pain. The PCC responded by reducing the opioid prescription and encouraging the wife to seek treatment for her own pain. The reduction was, in part, a recognition that the patient no longer needed as many pills if he was able to divert some to his wife.

\section{Insurance Constraints}

Concerns about insurance coverage also led to changes in pain management plans. Four of the event and action pairs revealed changes that were prompted by insurance concerns. In 1 case, a patient was doing well on celecoxib, but insurance stopped covering it, and the patient could not afford to pay for it out-of-pocket. The PCC switched to naproxen, but the patient did not respond to it as well as the celecoxib. Eventually, the patient started a new insurance plan and the PCC was able to resume celecoxib. In another case, a PCC tried to order pregabalin, but the insurance provider wanted the PCC to try other less expensive options first. Therefore, the PCC considered nortriptyline, but the patient worked in law enforcement and did not want any medications associated with depression on their medical record, leading to a further change to gabapentin.

"[Insurance] basically wanted me to try cheaper medications beforeband. So, I backed off. We originally did nortriptyline, but because be's a [law enforcement offcer], he did not want anything associated with a... mental diagnosis like depression. So, we stopped-we never actually used the nortriptyline... we immediately switched him over to the gabapentin."

-PCC 8, Pt 35

\section{Change in Guidelines, Laws, or Policies}

In some instances, pain management plans were changed in response to new opioid prescribing guidelines, laws, and institutional policies. This occurred in 3 of the event and action pairs. Examples of changes to pain management plans included reducing opioids, avoiding opioids and benzodiazepines, and using other modalities of pain management.

"So, when the [state] laws came down, I went to the conference downtown about it... Now the safety factor of giving benzos and opioids within last year with the CDC bas gotten even bigger. Somewhere, I want to say along [that timeline], I started weaning her off of benzos."

-PCC 5, Pt 26

"These days since we are cutting people, because of the new guidelines, you know, we're cutting people back on narcotics so much, I've been using a lot more of gabapentin, Lyrica, Cymbalta, that type of stuff, not just for neuropathy, but for even like osteoarthritis pain too."

-PCC 4, Pt 27

\section{Approaches to New Patients}

Some PCCs described rules of thumb that would trigger a change in pain management for new or transferred patients. This occurred in 2 of the event and action pairs. In both examples, PCCs described an opioid "threshold dose" for new patients. Opioids were reduced for new patients that exceed the threshold.

"Any patient of mine that is on 10 to 325, I've inherited, and I always try to wean them down to 1 of the lower doses."

-PCC 19, Pt 61

"I put her down to 30 and kind of took us away from that red flag dosing of narcotics and so I could get to know her better... we went ahead and did a pain contract even though the medications were not really bigh enough technically for a pain contract. I went ahead and did a pain contract to emphasize-and I kind of 
educated her about the dangers of the medications, risk of overdose and that it is habit forming, etc." -PCC 8, Pt 32

\section{Recommendations from Specialists}

Specialist recommendations can also lead to changes in pain management plans. One event and action pair revealed a change that resulted from a specialist recommendation. In the following example, a neuroradiology specialist recommended a referral to surgery. The PCC followed the recommendation but had concerns with the proposed treatment.

"So the neuroradiology people had suggested we have her see pain doctors because they do things like pain pumps and stuff like that so I put in that referral, a neurosurgery referral, though I hate to have her have more back surgery. . ."

-PCC 11, Pt 41

\section{Discussion}

Our study aimed to identify factors that influence PCCs to change existing pain management plans. The 7 factors we identified are depicted in Figure 1. In a related analysis, team members looked at the broader factors that influence sensemaking regard-

Figure 1. What leads to changes in existing chronic pain management plans? Seven themes emerged in our analysis of factors that influenced changes to chronic pain management plans based on interviews with primary care clinicians from 2016 to 2018 .

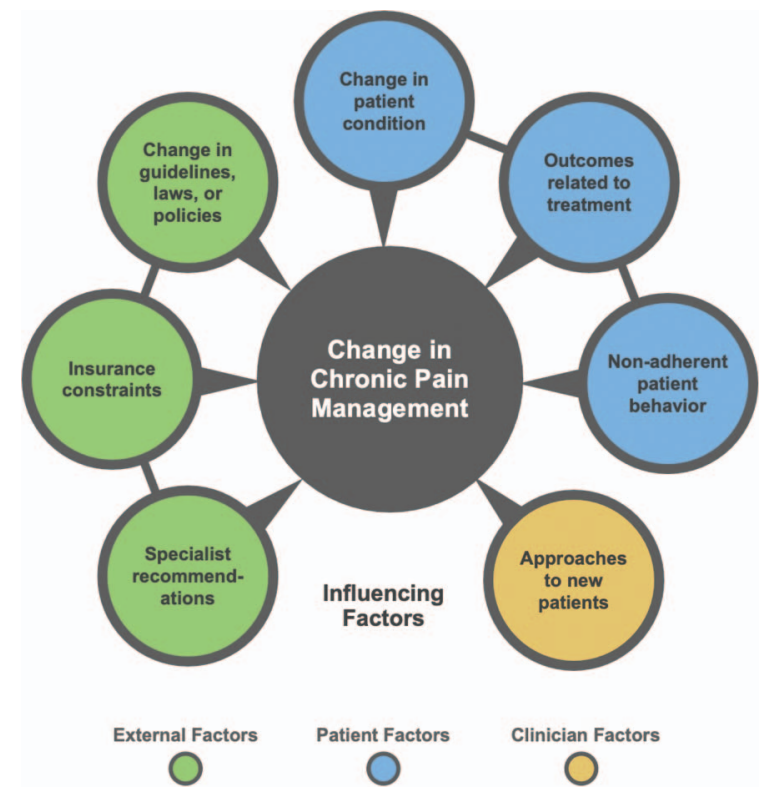

ing chronic pain. ${ }^{24}$ The sensemaking factors include external social/environmental factors, patient factors, and clinician characteristics. The factors we identify in this article fit neatly into those broader categories, lending support to our findings.

Chronic pain is notoriously difficult to treat due to frequent etiologic ambiguity and limited availability of efficacious and safe interventions. ${ }^{2,6}$ These difficulties may be exaggerated in the primary care context, where PCCs have limited training in pain management, minimal available time, and concerns about legal issues related to pain medications. ${ }^{3,7}$ New education and policy as well as point-of-care interventions, such as clinical process redesign, and clinical cognitive support tools may help clinicians navigate pain management more effectively and efficiently.

It is notable that when asked, none of the PCCs in our sample reported using cognitive support tools (or information from such tools) as a trigger for changing a pain management plan. This represents a potential leverage point as cognitive support tools could help PCCs identify and track factors that influence changes to pain management. We recommend future research to develop, implement, and assess the effectiveness of such innovations. As a step in that direction, we highlight an insight from each influencing factor and propose a related practice innovation (Table 1). These potential practice innovations are inspired by our findings. We do not intend to imply that our findings provide evidence regarding the utility of these broad innovations; rather, they are intended to inspire future research and exploration based on our understanding of the challenges described by PCCs and the decision strategies they report.

Our study has potential limitations. First, our sample consisted of PCCs, not pain specialists. However, PCCs treat most patients with chronic pain $^{3}$ and are arguably in greater need of cognitive support because they are less likely to receive formal training in pain management than pain specialists. Future investigations could look at the difference between what causes PCCs to change their pain management plans versus pain specialists. A second limitation is potential bias in selection of patients; it is possible that patients may have been selected to represent certain issues or their resolution. A third limitation is reliance on PCC recall of treatment events. Although we conducted our interviews within 3 days of the patient visit and en- 
Table 1. How can the Influencing Factors Identified in this Study Inform Practice Innovations? Key Insights and Potential Practice Innovations Inspired by the Themes that Emerged in our Analysis of Factors that Influenced Changes to Chronic Pain Management Plans Based on Interviews with Primary care Clinicians from 2016 to 2018

\begin{tabular}{|c|c|c|}
\hline Influencing Factor & Key Insight & Potential Practice Innovations \\
\hline Change in patient condition & $\begin{array}{l}\text { New observations about patients, such } \\
\text { as depression symptoms, and recent } \\
\text { falls can prompt changes to chronic } \\
\text { pain management. }\end{array}$ & $\begin{array}{l}\text { Innovations that monitor symptoms, } \\
\text { contraindications, and other } \\
\text { patient factors with the aim to } \\
\text { support PCCs in recognizing } \\
\text { when a change in pain } \\
\text { management may be warranted. }\end{array}$ \\
\hline Outcomes related to treatment & $\begin{array}{l}\text { Understanding treatment effectiveness } \\
\text { is a critical factor in chronic pain } \\
\text { management. }\end{array}$ & $\begin{array}{l}\text { Innovations that assist PCCs in } \\
\text { tracking the effectiveness of } \\
\text { treatments over time (e.g., } \\
\text { displays that correlate pain } \\
\text { outcomes with prescribed } \\
\text { treatments), including improved } \\
\text { definitions and measures of } \\
\text { effectiveness. }\end{array}$ \\
\hline $\begin{array}{l}\text { Non-adherent patient behavior } \\
\text { \& Approaches to new } \\
\text { patients }\end{array}$ & $\begin{array}{l}\text { Communication with patients is } \\
\text { essential. Conversations can reveal } \\
\text { non-adherent behavior. Upfront } \\
\text { discussions about pain goals can set } \\
\text { expectations for treatment. }\end{array}$ & $\begin{array}{l}\text { Innovations that collect and } \\
\text { synthesize hard-to-find data (such } \\
\text { as past treatments and rationale } \\
\text { for discontinuation), so PCCs } \\
\text { spend less time foraging for } \\
\text { information and more time } \\
\text { communicating and developing } \\
\text { relationships with patients. }\end{array}$ \\
\hline Insurance constraints & $\begin{array}{l}\text { Insurance constraints serve as a } \\
\text { decision-making factor in changing } \\
\text { pain management plans. However, } \\
\text { PCCs have little training, guidance, } \\
\text { or support with this task. }\end{array}$ & $\begin{array}{l}\text { Innovations that provide PCCs with } \\
\text { information about what } \\
\text { treatments are approved, the cost, } \\
\text { and other requirements such as } \\
\text { prior authorizations before } \\
\text { ordering. }\end{array}$ \\
\hline $\begin{array}{l}\text { Change in guidelines, laws, or } \\
\text { policies }\end{array}$ & $\begin{array}{l}\text { Changes in guidelines can lead to } \\
\text { changes in pain management for } \\
\text { individual patients. }\end{array}$ & $\begin{array}{l}\text { Innovations that facilitate accurate } \\
\text { interpretation of clinical practice } \\
\text { guidelines and help translate } \\
\text { those guidelines to simple, } \\
\text { actionable, point-of-care } \\
\text { guidance. }\end{array}$ \\
\hline Specialist recommendations & $\begin{array}{l}\text { Specialist input can influence pain } \\
\text { management, although PCCs may } \\
\text { not always agree with specialist } \\
\text { recommendations. }\end{array}$ & $\begin{array}{l}\text { Innovations in collaboration that } \\
\text { support PCCs in communicating } \\
\text { with other team members and } \\
\text { specialists. }\end{array}$ \\
\hline
\end{tabular}

PCCs, primary care clinicians.

couraged PCCs to use their electronic notes to verify historic information, there is risk that recall is incomplete. While most of the participants did consult the electronic health record (EHR) at some point in their interview, we do not know how many of the excerpts used in this analysis were derived from information obtained in the EHR. Although retrospective accounts are vulnerable to errors in recall, the Critical Decision Method technique is designed to improve accuracy of recall and provides insight into the context and rationale behind decision making, not easily obtained via other methods. ${ }^{22,25}$ Future investigations could validate our naturalistic observations with patient data. A fourth limitation involves the location and timing of our study. Our sample of US PCCs could have amplified a focus on opioids. In Europe, for instance, opioid treatment is relatively limited. ${ }^{26}$ Future investigations could look at what influences non-US PCCs to change their pain management plans. Notably, the influence of pharmaceutical companies did not emerge in our themes. This is surprising given research that links opioid marketing to increased prescribing. ${ }^{27}$ It is possible that marketing efforts influence the type of response but are not, by themselves, a trigger for action. Future investigations could track these themes over time to identify shifts in influencing factors. Future investigations should also include patients in the analysis process. 


\section{Conclusion}

Chronic pain management is difficult. Guidelines and training provide some direction, but PCCs must also use their own judgment and experience to treat patients. Our analysis sheds light on the factors that lead PCCs to change treatment plans for patients with chronic pain. An understanding these factors can inform the types of innovations that are needed to support PCCs in providing chronic pain care.

To see this article online, please go to: http://jabfm.org/content/ 33/1/42.full.

\section{References}

1. Gaskin DJ, Richard P. The economic costs of pain in the United States. J Pain 2012;13:715-24.

2. Harle CA, Bauer SE, Hoang HQ, Cook RL, Hurley RW, Fillingim RB. Decision support for chronic pain care: how do primary care physicians decide when to prescribe opioids? A qualitative study. BMC Family Practice 2015;16:48.

3. Committee on Advancing Pain Research Care Education, Institute of Medicine. Relieving pain in America: a blueprint for transforming prevention, care, education, and research. Washington, DC: The National Academies Press; 2011.

4. Leverence RR, Williams RL, Potter M, et al. Chronic non-cancer pain: a siren for primary care-A report from the PRImary Care MultiEthnic Network (PRIME Net). J Am Board Fam Med 2011;24:551-61.

5. Aldington D, Eccleston C. Evidence-based pain management: building on the foundations of Cochrane Systematic Reviews. AJPH Pain Management 2019;109:46-9.

6. Dowell D, Haegerich TM, Chou R. CDC guideline for prescribing opioids for chronic pain-United States, 2016. JAMA 2016;315:1624-45.

7. Abbo ED, Zhang Q, Zelder M, Huang ES. The increasing number of clinical items addressed during the time of adult primary care visits. J Gen Intern Med 2008;23:2058-65.

8. Chou R. 2009 Clinical Guidelines from the American Pain Society and the American Academy of Pain Medicine on the use of chronic opioid therapy in chronic noncancer pain: What are the key messages for clinical practice? Polskie Archiwum Medycyny Wewnetrznej 2009;119:469-477.

9. Manchikanti L, Abdi S, Atluri S, et al. American Society of Interventional Pain Physicians (ASIPP) Guidelines for responsible opioid prescribing in chronic non-cancer pain: Part 2- guidance. Pain Physician 2012;15:S67-S116.

10. Weiner SS, Weiser SR, Carragee EJ, Nordin M. Managing nonspecific low back pain: do nonclinical patient characteristics matter? Spine 2011;36:1987-94.
11. Finestone A, Raveh A, Mirovsky Y, Lahad A, Milgrom C. Orthopaedists' and family practitioners' knowledge of simple low back pain management. Spine 2009;34: 1600-3. Available from: http://ovidsp.ovid.com/ovidweb. cgi? $\mathrm{T}=\mathrm{JS} \& \mathrm{PAGE}=$ reference $\& \mathrm{D}=$ emed $9 \& \mathrm{NEWS}=$ $\mathrm{N} \& A N=2009380439$.

12. Williams CM, Maher CG, Hancock MJ, et al. Low back pain and best practice care: A survey of general practice physicians. Arch Intern Med 2010;170: 271-7.

13. Dey P, Simpson CW, Collins SI, et al. Implementation of RCGP guidelines for acute low back pain: a cluster randomised controlled trial. Br J Gen Pract 2004;54:33-7.

14. Morasco BJ, Duckart JP, Dobscha SK. Adherence to clinical guidelines for opioid therapy for chronic pain in patients with substance use disorder. J Gen Intern Med 2011;26:965-71.

15. Østbye T, Yarnall KS, Krause KM, Pollak KI, Gradison M, Michener JL. Is there time for management of patients with chronic diseases in primary care? Ann Fam Med 2005;3:209-14.

16. Cavalier J Jr, Hampton SB, Langford R, Symes L, Young A. The influence of race and gender on nursing care decisions: a pain management intervention. Pain Manag Nurs 2018;19:238-45.

17. Chen L, Houghton M, Seefeld L, Malarick C, Mao J. Opioid therapy for chronic pain: physicians' attitude and current practice patterns. J Opioid Manag 2011;7:267-76.

18. Green CR, Wheeler JR, LaPorte F. Clinical decision making in pain management: contributions of physician and patient characteristics to variations in practice. J Pain 2003;4:29-39.

19. Hirsh AT, Hollingshead NA, Ashburn-Nardo L, Kroenke K. The interaction of patient race, provider bias, and clinical ambiguity on pain management decisions. J Pain 2015;16:558-68.

20. Hollingshead NA, Matthias MS, Bair MJ, Hirsh AT. Impact of race and sex on pain management by medical trainees: a mixed methods pilot study of decision making and awareness of influence. Pain Med 2015;16:280-90.

21. Turk DC, Okifuji A. What factors affect physicians' decisions to prescribe opioids for chronic noncancer pain patients? Clin J Pain 1997;13;330-336.

22. Crandall B, Klein G, Hoffman RR. Working minds: a practitioner's guide to cognitive task analysis. Cambridge, MA: MIT Press; 2006.

23. Barbour RS. Checklists for improving rigour in qualitative research: a case of the tail wagging the dog? BMJ 2001;322:1115-7.

24. Militello LG, Anders S, Downs SM, et al. Understanding how primary care clinicians make sense of chronic pain. Technol Work 2018;20:575-584.

25. Crandall B. A comparative study of think-aloud and critical decision knowledge elicitation methods. SIGART Newsletter 1989;108:144-6. 
26. The opioid epidemic: what can we learn from Europe? (August 14, 2018). Cleveland Clinic, Department of Pain Management, Consult QD. Available from: https://consultqd.clevelandclinic.org/theopioid-epidemic-what-can-we-learn-from-europe/.
27. Hadland SE, Cerdá M, Li Y, Krieger MS, Marshall BDL. Association of pharmaceutical industry marketing of opioid products to physicians with subsequent opioid prescribing. JAMA Intern Med 2018; 178:861-3. 